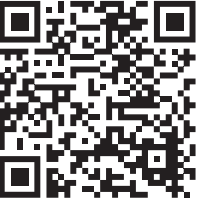

* Unidad de Medicina Familiar Núm. 24, «lgnacio García Téllez», Instituto Mexicano del Seguro Social. Médico de Medicina Familiar. Nayarit, México. ₹ Unidad de Medicina Familiar Núm. 24, «lgnacio García Téllez», Instituto Mexicano del Seguro Social. Coordinador Clínico de Investigación y Educación. Nayarit, México. § Universidad Autónoma de Nayarit. Especialista en Pediatría Médica. Profesor en la Unidad Académica de Medicina. Nayarit, México.

" Tecnológico Nacional de México, Campus Tepic. Profesor investigador en Ciencias en Alimentos. Nayarit, México.

Correspondencia: VMZC vzamora@ittepic.edu.mx Conflicto de intereses: Los autores declaramos no tener intereses personales, comerciales, financieros o económicos directos o indirectos ni conflictos de interés de cualquier índole, que pudieran representar un sesgo para la información presentada en este artículo.

Citar como: AlarcónDomínguez EE, VelascoGonzález LE, MedinaCarrillo L, Zamora-Gasga VM. Influencia de factores perinatales y alimentarios sobre el desarrollo de sobrepeso y obesidad en lactantes. Rev CONAMED. 2020; 25(2): 66-74. doi: $10.35366 / 94389$

Financiamiento: Ninguno.

Recibido: 13/08/2019 Aceptado: 30/10/2019

\section{Influencia de factores perinatales y alimentarios sobre el desarrollo de sobrepeso y obesidad en lactantes}

\author{
Influence of perinatal and dietary factors on the \\ development of overweight and obesity in infants \\ Edith Efrén Alarcón-Domínguez, ${ }^{*}$ Luisa Elizabeth Velasco-González, ${ }^{\ddagger}$ \\ Leopoldo Medina-Carrillo, ${ }^{\S}$ Víctor Manuel Zamora-Gasgall
}

\section{RESUMEN}

Introducción: El sobrepeso y la obesidad infantil representan un problema de salud pública y se ha reportado su prevalencia de 8.2 y $6.3 \%$ respectivamente, en lactantes mexicanos. Algunas investigaciones incluyen como factores de riesgo para el desarrollo de sobrepeso y obesidad: alto peso al nacer, aumento acelerado de peso infantil y la ausencia de lactancia materna durante el primer año de vida. En Nayarit, no existen reportes de las causas asociadas con esta patología. Objetivo: Evaluar la influencia de los factores perinatales y alimentarios sobre el desarrollo de sobrepeso y obesidad en lactantes en una unidad médica de primer nivel de atención del sector público en Tepic, Nayarit. Métodos: Se trató de un estudio observacional, retrospectivo y transversal de casos (de sobrepeso-obesidad; $n=30$ ) y controles (con normopeso; $n=60$ ). Se evaluó el estado nutricional actual, peso al nacer, tipo de alimentación, duración de la lactancia materna, edad y alimento de inicio de la alimentación complementaria. El análisis de datos se realizó utilizando la prueba t Student, el estadístico $\chi^{2}$ y un análisis de regresión logística con el programa Statistica versión $12(\alpha=0.05)$. Resultados: En la variable de prevalencia de lactancia materna, se observó una diferencia de 10\% mayor en el grupo control (23.33\%) que en el grupo de casos (13.33\%); sin embargo, la alimentación complementaria mixta fue predominante en los infantes (> 70\%). El principal alimento de inicio fueron las verduras con $50 \%$ para los casos y $38.33 \%$ para los controles. El grupo de casos presentó menor talla $(79.56 \mathrm{~cm})$ que el grupo control $(82.40 \mathrm{~cm})$, y la duración de la lactancia materna se asoció como un factor protector para el desarrollo de sobrepeso y obesidad (razón de momios $=0.896$ ). Conclusiones: Los resultados de este trabajo muestran la importancia de incrementar el periodo de la lactancia materna, ya sea exclusiva o mixta, como una posible estrategia para disminuir los problemas de peso excesivo en los infantes.

Palabras clave: Primer nivel de atención, lactancia materna, alimentación complementaria, factor de riesgo, obesidad infantil.

\section{ABSTRACT}

Introduction: Child overweight and obesity represent a health problem public and a prevalence of 8.2 and 6.3\%, respectively, has been reported in Mexican infants. Some research includes as risk factors for the development of overweight and obesity, high birth weight, rapid increase in infant weight, the absence of breastfeeding during the first year of life, among others. In Nayarit, there are no reports of the causes associated with this pathology. Objective: To assess the influence of perinatal and dietary factors on the development of overweight and obesity in infants in a first-level medical unit of public sector care in Tepic, Nayarit. Methods: An observational, retrospective, cross-sectional study of cases (overweight-obesity, $n=30$ ) and controls (normal weight, $n=60$ ) was performed. The current nutritional status, weight at birth, feeding type, duration of breastfeeding, age and start food of the complementary feeding were evaluated. Data analysis was performed using the student $t$ test, the $\chi^{2}$ test and a logistic 
regression analysis with the STATISTICA version $12(\alpha=0.05)$. Results: In the prevalence of breastfeeding, a $10 \%$ greater difference was observed in the control group $(23.33 \%)$ than in the case group (13.33\%), however, mixed complementary feeding was predominant in infants (> 70\%). The main starting food was vegetables with $50 \%$ for cases and $38.33 \%$ for controls. The case group presented smaller height $(79.56 \mathrm{~cm})$ than the control group $(82.40 \mathrm{~cm})$ and the duration of breastfeeding was associated as a protective factor towards the development of overweight and obesity (Odds ratio $=0.896$ ). Conclusions: The results of this work show the importance of increasing the period of breastfeeding, either exclusive or mixed, as a possible strategy to reduce problems of excessive weight in infants.

Keywords: First level of attention, breastfeeding, complementary feeding, risk factor, childhood obesity.

\section{INTRODUCCIÓN}

La lactancia materna (LM) es la base de la alimentación durante los inicios de la infancia. Según la Organización Mundial de la Salud (OMS), se recomienda la LM durante la primera hora después del nacimiento.' También, se sugiere mantenerla de forma exclusiva hasta los seis meses, y continuar junto con otros alimentos hasta los dos años de vida. ${ }^{2}$ En México, la duración de la lactancia materna es de cerca de 10 meses, cifra estable entre 1999 y 2012 (9.7 y 10.2 meses, respectivamente). Sin embargo, entre 2006 y 2012, el porcentaje de LM exclusiva en menores de seis meses disminuyó de 22.3 a $14.5 \%$ en la zona urbana y de 36.9 a $18.5 \%$ en el medio rural. ${ }^{3}$

Por una parte, la LM tiene múltiples beneficios y se ha reportado como un factor protector contra el desarrollo de sobrepeso y obesidad en la infancia y la adolescencia. ${ }^{4}$ Por la otra, a la introducción de otros alimentos distintos de la leche materna se le denomina alimentación complementaria (AC). El inicio de la AC depende de aspectos nutricionales, digestión y absorción adecuada de nutrientes, control cefálico, sedestación, uso de la musculatura masticatoria, dentición, incremento de las percepciones sensoriales, extinción del reflejo de extrusión y discriminación de nuevas texturas, sabores, olores y temperaturas. ${ }^{5}$ La manera de alimentarse y la preferencia o rechazo hacia determinados alimentos están relacionadas por el aprendizaje en los primeros cinco años de vida. Así, el infante incorpora la mayoría de los hábitos y prácticas alimentarias antes de esa edad. ${ }^{6}$

La recomendación establece que la AC debe iniciarse a partir de los seis meses; al respecto, hay evidencia suficiente que demuestra que no es hasta ese momento cuando los beneficios potenciales de introducir alimentos comienzan a superar a los riesgos.7 Uno de los principales riesgos es el desarrollo de sobrepeso y obesidad en los lactantes. En este sentido, la prevalencia de sobrepeso y obesidad en preescolares mexicanos disminuyó en la última década. Los resultados muestran que, entre 2012 y 2016 en la población en edad preescolar, el exceso de peso pasó de 31.8 a 23.5\% en niñas y de 35.2 a $21.2 \%$ en niños. ${ }^{8}$ Además, se han informado cifras de sobrepeso y obesidad de 23.7 y $7.9 \%$ para preescolares niños y de 8 y $24 \%$ para preescolares niñas. ${ }^{9}$ Por su parte, Wollenstein-Seligson e IglesiasLeboreiro ${ }^{10}$ reportaron una prevalencia general de sobrepeso y obesidad de 8.2 y $6.3 \%$ en lactantes, respectivamente (de uno a dos años).

En Nayarit, la prevalencia de sobrepeso y obesidad en menores de cinco años fue de 10.7\%, con una distribución para las localidades urbanas de $9.5 \%$ y para las rurales de $13.3 \% .^{3}$ Algunos reportes indican que la obesidad pregestacional y la alimentación con fórmula láctea exclusiva incrementan hasta $64.38 \%$ la probabilidad de desarrollar sobrepeso y obesidad en lactantes mexicanos a los 12 meses de edad." Varios factores de riesgo durante los primeros mil días se asociaron consistentemente con la obesidad infantil. Entre éstos, principalmente se encontraron un índice de masa corporal (IMC) materno más alto antes del embarazo, la exposición prenatal al tabaco, el aumento de peso gestacional excesivo materno, la presencia de diabetes gestacional, alto peso al nacer y aumento acelerado de peso infantil.12 En este sentido, Larqué y Labayén ${ }^{13}$ incluyen la ausencia de lactancia materna durante el primer año de vida, el alto consumo de proteínas y azúcares en los infantes, obesidad materna y paterna en la concepción, desnutrición materna, alta ingesta materna de azúcares y una corta duración del sueño infantil como factores de riesgo para el desarrollo de obesidad en lactantes. 
Es así que la obesidad infantil debe abordarse desde una perspectiva multicausal en la que el individuo como los padres, el medio familiar o el ambiente en general puedan favorecer su aparición." Por tanto, es necesaria la evaluación de los indicadores de crecimiento que permitan determinar problemas en estado nutricional del infante, ya sea por estados carenciales o por exceso, que requirieran de la toma de decisiones y acciones para mejorar su condición. A partir de ello, se estableció como objetivo del presente trabajo el evaluar la influencia de factores de riesgo perinatales y alimentarios sobre el desarrollo de sobrepeso y obesidad en lactantes adscritos a la Unidad de Medicina Familiar Núm. 24 «lgnacio García Téllez» de Tepic, Nayarit.

\section{DESCRIPCIÓN DEL MÉTODO}

Se realizó un estudio observacional, retrospectivo y transversal de casos (prevalentes) y controles. El tamaño de la muestra se calculó con el programa Epidat versión 4.1, utilizando una población de 1,765 lactantes de 12 a 24 meses (estadística tomada del archivo clínico de la propia unidad hasta junio de 2015), un nivel de confianza de 95\%, una potencia de 80\%, una proporción de 1:2 entre el número de casos y controles, una frecuencia esperada de la exposición principal en el grupo de control (destete precoz) de 40\% y una razón de momios de 2 , acorde con datos de la literatura. ${ }^{14}$

El número de casos fue de 27, pero se consideró un 10\% más por reposición de pérdidas, para un total de 30 casos y 60 controles. La selección de los individuos de ambos grupos se realizó por un muestreo por cuota hasta cumplir con el tamaño de la muestra previamente calculada, considerando aquellos pacientes en el turno matutino y el vespertino. Los pacientes fueron elegidos de la consulta externa de medicina familiar y de los módulos de PrevenIMSS, previo consentimiento informado y autorización de los padres durante el periodo enero a julio de 2017.

A los infantes se les determinó su peso y talla, utilizando para ello una báscula mecánica de columna con estadiómetro, con peso máximo de $140 \mathrm{~kg}$. Los participantes fueron clasificados en dos grupos de acuerdo con las curvas de los percentiles del índice de masa corporal (IMC) para la edad, de acuerdo con lo establecido por la Organización Mundial de la Salud (OMS)..$^{15}$ Así, los criterios de inclusión para el grupo de casos ( $n=30$ ) fueron: 1) edad de 12 a 24 meses y 2) presentar sobrepeso y obesidad (percentil > 85). Los criterios de inclusión en el grupo de control ( $n=60)$ fueron: 1 ) edad de 12 a 24 meses y 2) presentar normopeso (percentil entre 15 a 85). El cálculo de los percentiles se realizó utilizando la aplicación WHO Anthro.Ink, versión 3.2.2 (https://www.who.int/childgrowth/software/en/).

En el estudio, se consideraron como criterios de exclusión la presencia de enfermedades congénitas, inmunológicas, oncológicas o infecciosas. Finalmente, como criterio de eliminación, se consideró a los infantes cuyas encuestas mostraron datos antropométricos o perinatales incompletos. Para la evaluación de los factores de riesgos asociados con el desarrollo de sobrepeso y obesidad, se realizó una historia clínica, expediente clínico y la revisión de la Cartilla Nacional de Salud. La recolección de los datos se realizó a través de un cuestionario a la madre de los infantes, el cual se administró por el personal capacitado mediante preguntas con opción múltiple a fin de determinar las siguientes variables: género, edad de inicio de la alimentación complementaria, peso al nacer (obtenido de la Cartilla Nacional de Salud), el tipo de alimento consumido al inicio de la alimentación complementaria y alimentación al seno materno de manera exclusiva o la alimentación con fórmula láctea o mixta (Anexo 7).

Para el análisis univariante, las variables continuas se representaron como la media \pm desviación estándar. Las comparaciones entre los parámetros antropométricos (peso al nacer, edad, talla peso y percentil actual), tiempo de lactancia y los grupos de estudio se realizaron utilizando la prueba t de Student para grupos independientes. Las variables categóricas se expresaron como la frecuencia absoluta y el porcentaje. Se determinaron las asociaciones entre las variables categóricas (género, tipo de lactancia, tipo de alimentación y edad de inicio de alimentación complementaria) y los grupos de estudio (casos y controles) mediante tablas de contingencia, utilizando para ello la prueba $\chi^{2}$. El análisis multivariante de los factores asociados con la aparición de sobrepeso y obesidad en los infantes se realizó mediante una regresión logística binaria, incluyendo las variables categóricas y continuas 
hasta la obtención del modelo final. El análisis de los datos se realizó mediante el programa Statistica versión 12 (StatSoft, Inc. Tulsa, USA), con un nivel de significación de 0.05 .

\section{Consideraciones éticas}

En el presente estudio, se cumplió con los principios éticos de las investigaciones en humanos establecidos en la Declaración de Helsinki y de acuerdo con el Reglamento de la Ley General de Salud en Materia de Investigación para la Salud. El diseño de esta investigación fue discutido en el Consejo Científico y aprobado por el Comité de Ética del Hospital con número de asignación 2017-1801-5.

\section{RESULTADOS}

En la Tabla 7, se muestra la relación entre los parámetros antropométricos, el tiempo de lactancia materna y los grupos estudiados. Se determinó que las variables peso al nacer y la edad no tuvieron diferencia estadística ( $p>0.05$ ). El peso al nacer medio para el grupo de casos fue de 3,169.66 g y para el control fue de 3,145.25 g. La edad de los infantes se encontró entre los 18.63 y 20.33 meses. Asimismo, se observó que en el peso actual, talla, IMC, percentil y el tiempo de la lactancia se encontraron diferencias significativas entre los grupos $(p<0.05)$. El peso actual del grupo caso fue mayor que el grupo control por una diferencia de 1,746.66 g. Un aspecto importante, fue la notable diferencia entre los casos y controles con respecto a la talla (79.56 y $82.40 \mathrm{~cm}$, respectivamente) y la duración de la lactancia materna (6.33 y 9.76 meses, respectivamente). Además, para el IMC y el percentil ( $\mathrm{p}$ $<0.05$ ), los valores en los casos (20 kg/ $/ \mathrm{m}^{2}$ y $97.29 \%$, respectivamente) fueron superiores comparados con los controles $\left(16.02 \mathrm{~kg} / \mathrm{m}^{2}\right.$ y $49.45 \%$, respectivamente).

Entre los 90 lactantes estudiados, 30 casos y 60 controles; 54 eran varones (60\%) y 36 eran mujeres (40\%). De los 54 varones, 17 eran casos (31.48\%) y 37 eran controles (68.52), y de las 36 lactantes del grupo femenino se reportaron 13 casos (36.11\%) y 23 controles (63.89\%). En la Figura 1, se muestra la relación entre el género y los grupos de estudio. No se determinó una relación entre el género y el estado nutricional de los lactantes ( $p>0.05)$. Se observó una diferencia de 5\% mayor entre el grupo casos con respecto a los controles en el género femenino en comparación con el género masculino, que fue 5\% menor. También no se evidenció una relación entre el tipo de lactancia y el estado nutricional $(p>0.05)$.

Con respecto a lactancia materna, se notó una diferencia de $10 \%$ mayor en el grupo control con respecto al grupo de casos (Figura 2). Mientras que para la fórmula láctea, se observó más de 13\% para el grupo de casos que el control. Con respecto a la lactancia mixta, los valores discreparon por poco más de 3\%, siendo esta opción la que mayor prevalencia mostró en ambos grupos ( $\geq 70 \%)$. A razón de la duración de la lactancia materna, sin importar

Tabla 1: Evaluación antropométrica y tiempo de lactancia por grupo de estudio en infantes adscritos a la Unidad de Medicina Familiar Núm. 24 «Ignacio García Téllez» del IMSS de Tepic, Nayarit.*

\begin{tabular}{lcc}
\hline & Caso & Control \\
\hline Peso al nacer $(\mathrm{g})$ & $3,169.66 \pm 541.43 \mathrm{a}$ & $3145.25 \pm 534.65 \mathrm{a}$ \\
Edad (meses) & $20.33 \pm 4.42 \mathrm{a}$ & $18.63 \pm 4.84 \mathrm{a}$ \\
Peso actual $(\mathrm{g})$ & $12,663.33 \pm 1897.63 \mathrm{a}$ & $10916.67 \pm 1532.63 \mathrm{~b}$ \\
Talla $(\mathrm{cm})$ & $79.56 \pm 5.32 \mathrm{a}$ & $82.40 \pm 5.87 \mathrm{~b}$ \\
IMC $\left(\mathrm{kg} / \mathrm{m}^{2}\right)$ & $20.00 \pm 2.43 \mathrm{a}$ & $16.02 \pm 0.88 \mathrm{~b}$ \\
Percentil $(\%)$ & $97.29 \pm 4.04 \mathrm{a}$ & $49.45 \pm 26.22 \mathrm{~b}$ \\
Tiempo de lactancia (meses) & $6.33 \pm 6.27 \mathrm{a}$ & $9.76 \pm 6.01 \mathrm{~b}$ \\
\hline
\end{tabular}

*Los valores fueron expresados como la media \pm desviación estándar para casos $(n=30)$ y controles $(n=60)$. Letras diferentes entre filas muestran diferencia significativa entre las variables por grupo de estudio $(p<0.05)$. 


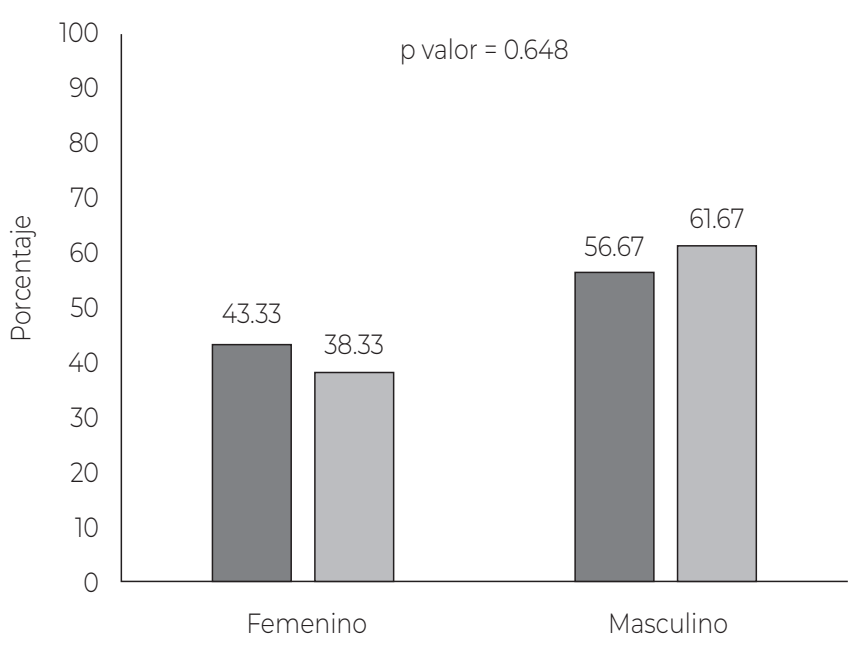

Figura 1: División por sexo del grupo de casos con sobrepesoobesidad ( $\square$ ) y del grupo control con normopeso (ㅁ).

si ésta fue mixta o exclusiva, se podría establecer una relación proporcional entre el grupo control y el tiempo de duración de la lactancia materna ( $\mathrm{P}$ $<0.05$, Tabla 7). Acerca del tipo de alimentación, se analizó un total de seis grupos de alimentos (Figura 3), de los cuales no se determinó relación entre el tipo de alimentación y el estado nutricional de los lactantes ( $p>0.05)$. El grupo de verduras fue el alimento principal en la alimentación complementaria de los infantes. Respecto a este alimento, se observó una diferencia porcentual 11.67 puntos más en el grupo de casos (50\%) que en el grupo control (38.33\%). En la Figura 4 se muestran los resultados entre la relación del periodo de inicio de la alimentación complementaria y los grupos evaluados.

La edad de inicio de alimentación complementaria fue correcta en cerca de $58.33 \%$ de los controles y de $46.67 \%$ de los casos. También se observó que $36.67 \%$ de los casos inició su alimentación complementaria antes de los seis meses. A pesar de estas diferencias, no se evidenció una relación significativa entre la edad de inicio de la alimentación complementaria y los grupos evaluados ( $p>0.05)$.

El análisis multivariante de regresión logística respecto al desarrollo de sobrepeso y obesidad $(Y)$ durante el estudio se muestra en la Tabla 2. Los factores de riesgo $(X i)$ incluidos en la ecuación fueron $X_{1}$ : peso al nacer $(\mathrm{kg}) ; X_{2}$ : edad (meses); $X_{3}$ : género femenino; $X_{4}$ : lactancia materna exclusiva; $X_{5}$ : tiempo de lactancia materna (meses); $X_{6}$ : correcta edad de inicio de alimentación complementaria (seis meses), y $X_{7}$ : verdura como alimento complementario.

La ecuación general de regresión logística del modelo de predicción fue la siguiente:

$$
\begin{gathered}
P(Y)=1 /\left(1+\exp .\left(2.836-0.443 X_{1}-0.091 X_{2}-\right.\right. \\
\left.0.055 X_{3}-0.373 X_{4}+0.109 X_{5}+0.819 X_{6}-0.419 X_{7}\right)
\end{gathered}
$$

De los factores alimentarios, se evidenció que el tiempo de lactancia materna $\left(X_{5}\right)$ fue significativo para el modelo. Esta variable presenta un efecto protector hacia el desarrollo de sobrepeso y obesidad en los infantes con una razón de momios (RM) de 0.896. Además, la correcta edad de inicio de la alimentación complementaria se mostró como un factor protector $(R M=0.441)$, pero no fue estadísticamente significativa ( $p=0.117)$. El resto de las variables no fueron significativas para el modelo $(p>0.05)$.

\section{DISCUSIÓN}

En la actualidad, es bien conocida la prevención que tiene la lactancia materna para evitar el desarrollo de sobrepeso y obesidad. ${ }^{16}$ Desde el periodo pregestacional y gestacional, factores como la obe-

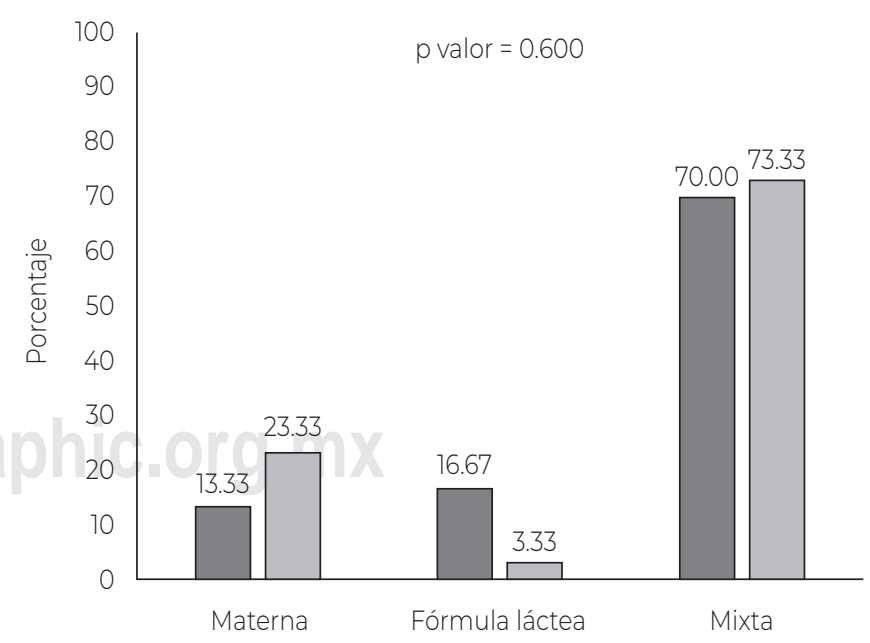

Figura 2: Tipo de lactancia en el grupo de casos con sobrepeso-obesidad ( $\square$ ) y del grupo control con normopeso (口). 


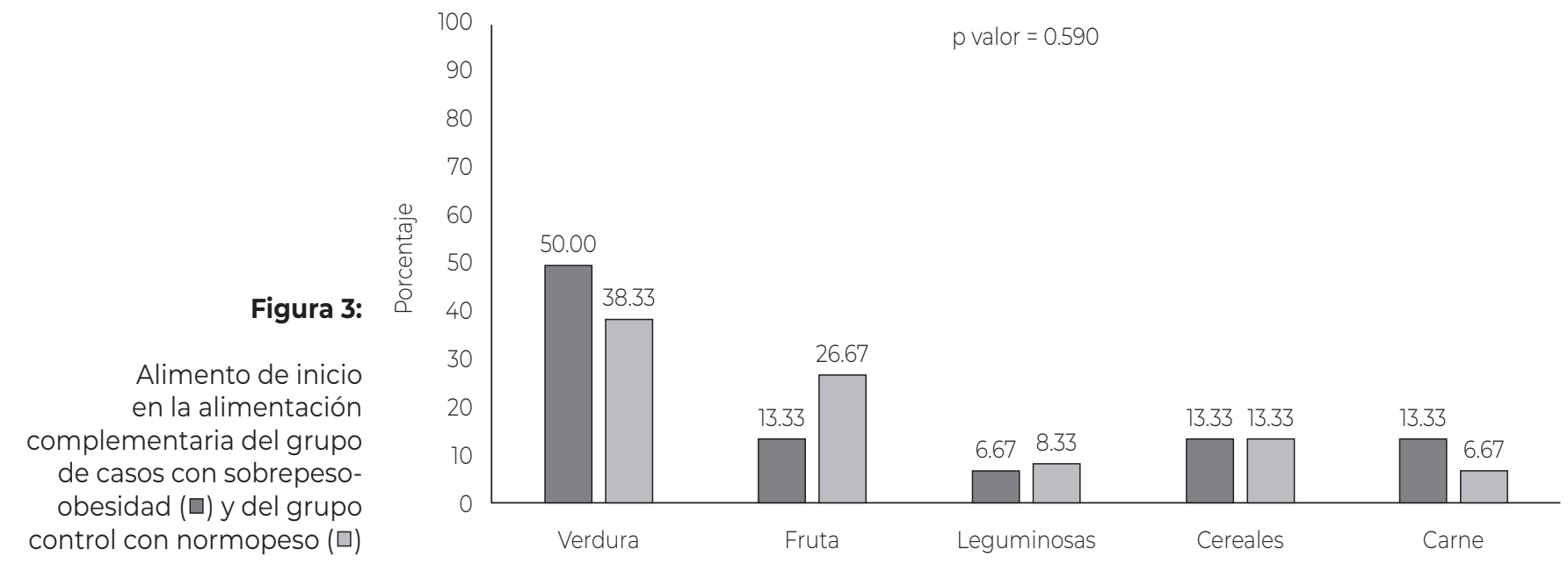

sidad materna, el incremento de peso gestacional de la madre, la multiparidad, el peso al nacer y la prematurez son determinantes para una mayor susceptibilidad de sobrepeso u obesidad infantil. ${ }^{7}$ Por esta razón, el presente trabajo demostró que el principal factor de riesgo para el desarrollo de sobrepeso y obesidad fue la disminución en el tiempo de duración de la lactancia materna. Además, los lactantes con normopeso presentaron mayor talla comparados con el grupo con sobrepeso y obesidad (diferencia de 3.5 puntos porcentuales). Esto sugiere que el lactante que es alimentado al seno materno durante mayor tiempo durante sus

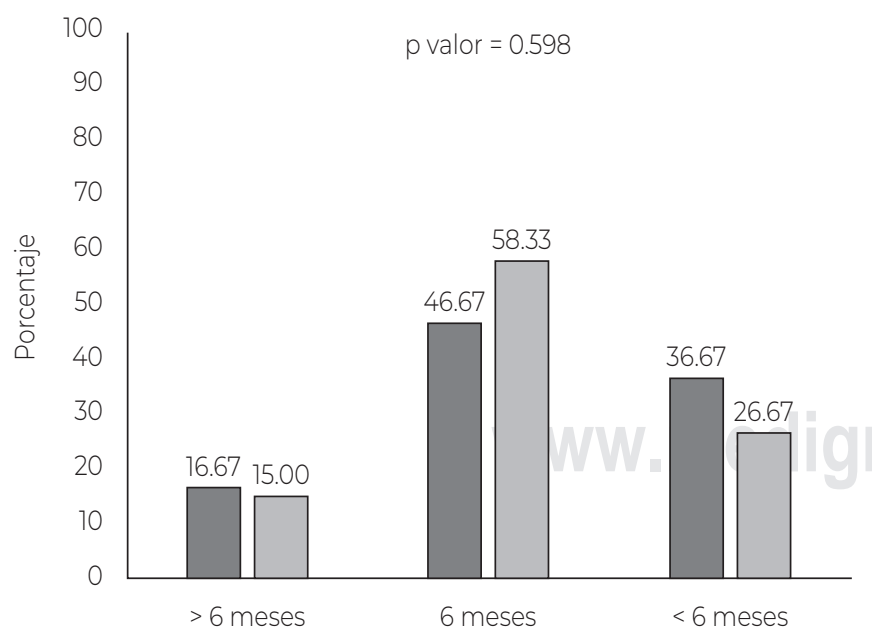

Figura 4: Edad de inicio de la alimentación complementaria en el grupo de casos con sobrepeso-obesidad ( $\square$ ) y del grupo control con normopeso ( $\square$ ). primeros dos años de vida refleja un estado nutricional adecuado con mayor estatura.

En este trabajo, el género, la forma de alimentación (lactancia materna exclusiva o mixta), el periodo y el grupo de alimento con la que el infante inició su alimentación complementaria no fueron factores de riesgo para el desarrollo de sobrepeso y obesidad. Otros factores que no fueron evaluados, como las prácticas alimentarias de los padres durante la infancia temprana y la introducción de alimentos sólidos, han sido propuestos como la clave determinante capaz de modificar la obesidad infantil. ${ }^{18}$ En este sentido, Reyes y Reyes ${ }^{19}$ sugieren que la introducción de alimentos sólidos antes de los cuatro meses de edad se asocia con una mayor ganancia de peso durante la infancia. Esto no concuerda con los hallazgos encontrados en esta investigación, ya que nuestros resultados sugieren que el tipo de alimentación, ya sea leche materna exclusiva o alimentación mixta (leche materna más alimentación complementaria), no se asocia con el desarrollo de sobrepeso y obesidad en los lactantes. Esto podría relacionarse con que más de $80 \%$ de los lactantes recibe la alimentación complementaria a los seis o más meses de vida, lo que coincide con lo recomendado por la OMS. Además, en el presente estudio, se determinó un efecto protector de la lactancia materna contra el sobrepeso directamente proporcional al tiempo de duración; estos resultados son similares a los reportados en otros trabajos de investigación. ${ }^{20}$

Por otro lado, la Encuesta Nacional de Nutrición y Salud refleja que en los lactantes menores de seis 
Tabla 2: Análisis de regresión logística binaria para el desarrollo de sobrepeso y obesidad en lactantes de la Unidad de Medicina Familiar Núm. 24 «Ignacio García Téllez» del IMSS de Tepic, Nayarit.

\begin{tabular}{|c|c|c|c|c|c|c|c|}
\hline & \multirow[b]{2}{*}{$\begin{array}{l}\text { Y: Variable binaria } \\
\text { (0 control; } 1 \text { caso) }\end{array}$} & \multirow[b]{2}{*}{$\beta$} & \multirow[b]{2}{*}{$\begin{array}{l}\text { Error } \\
\text { estándar }\end{array}$} & \multirow[b]{2}{*}{$p$} & \multirow[b]{2}{*}{$\mathrm{RM}$} & \multicolumn{2}{|c|}{ IC del 95\%, para RM } \\
\hline & & & & & & Inferior & Superior \\
\hline \multicolumn{8}{|c|}{$\mathrm{Xn}$} \\
\hline 1 & Peso al nacer (kg) & 0.443 & 0.455 & 0.333 & 1.557 & 0.63 & 3.847 \\
\hline 2 & Edad (meses) & 0.091 & 0.060 & 0.134 & 1.096 & 0.972 & 1.235 \\
\hline 3 & Género femenino & 0.055 & 0.526 & 0.917 & 1.057 & 0.371 & 3.011 \\
\hline 4 & $\begin{array}{l}\text { Lactancia materna } \\
\text { exclusiva }\end{array}$ & 0.373 & 0.823 & 0.652 & 1.452 & 0.282 & 7.469 \\
\hline 5 & $\begin{array}{l}\text { Tiempo de lactancia } \\
\text { materna (meses) }\end{array}$ & -0.109 & 0.050 & 0.032 & 0.896 & 0.811 & 0.99 \\
\hline 6 & $\begin{array}{l}\text { Correcta edad de inicio } \\
\text { de alimentación com- } \\
\text { plementaria }\end{array}$ & -0.819 & 0.517 & 0.117 & 0.441 & 0.158 & 1.232 \\
\hline 7 & $\begin{array}{l}\text { Verdura como alimento } \\
\text { complementario }\end{array}$ & 0.419 & 0.510 & 0.414 & 1.52 & 0.551 & 4.195 \\
\hline 8 & Constante & -2.836 & 1.954 & 0.151 & 0.059 & 0.001 & 2.863 \\
\hline
\end{tabular}

$\mathrm{Y}=$ estado nutricional; $\mathrm{Xn}$ = factor de riesgo; $\mathrm{IC}$ = Intervalo de confianza; $\mathrm{RM}=$ Razón de momios

meses la práctica de lactancia materna exclusiva mostró un notable deterioro al pasar de $22.3 \%$ en 2006 a 14.5\% en 2012. ${ }^{3}$ Este descenso, sumado al incremento en la utilización de fórmulas lácteas para alimentar a los infantes (evidenciado en los resultados de esta investigación), ha sido asociado con el desarrollo de sobrepeso y obesidad, debido al alto contenido en proteínas que este alimento presenta. ${ }^{20}$ En cuanto a las limitaciones del estudio, la sobreposición del promedio del peso actual de los casos y los controles, aunado a la desviación estándar, puede crear dificultades para replicar resultados en otras poblaciones. Además, se puede dar lugar a un sesgo de recuerdo, es decir, a la memoria de las madres respecto al inicio de la lactancia y el tipo de alimentación. Por tanto, es necesario confirmar los resultados en esta población, considerando el incremento en el tamaño muestral para ambos grupos.

Por último, pese a los grandes esfuerzos por parte de los servicios de salud y políticas gubernamentales, aún no se ha logrado que la práctica de la alimentación con leche materna sea uniforme. En México, la Estrategia Nacional de Lactancia Materna tiene como objetivos específicos trabajar coordinadamente y de forma interinstitucional para desarrollar las acciones, el seguimiento de los resultados y el fortalecimiento de las capacidades institucionales que contemplen los beneficios de la lactancia materna. Sin embargo, de acuerdo con varios expertos, ${ }^{21}$ para que esta estrategia sea exitosa, debe estar basada en la experiencia obtenida por otros países en las áreas de promoción, protección y a poyo a la lactancia que busque mejorar la salud materno-infantil.

\section{CONCLUSIÓN}

En este trabajo se estableció que más de 80\% de los infantes inicia la alimentación complementaria a los seis o más meses cumplidos, tal como lo establecen las normas internacionales. Por tanto, la alimentación con leche materna exclusiva o mixta no se relacionó directamente con el desarrollo de 
sobrepeso y obesidad. Además, se evidenció que los lactantes iniciaron la alimentación complementaria principalmente con verduras, y que la presencia de sobrepeso se asoció con una menor estatura. Finalmente, una mayor duración de la lactancia, ya sea mixta o exclusiva, proporcionó un efecto protector ante el desarrollo del sobrepeso y la obesidad. Estos resultados no son concluyentes, ya que pueden existir sesgos de recuerdo y es necesario realizar estudios en muestras de mayor tamaño que confirmen los hallazgos encontrados en la presente investigación.

Los resultados de este trabajo muestran que es de gran importancia el incrementar el periodo de la lactancia materna, pues esto puede servir como una posible estrategia para disminuir los problemas de peso excesivo. Particularmente, podría ser utilizada para ayudar al médico de primer nivel de atención en la identificación de los pacientes con mayor riesgo de desarrollo de sobrepeso y obesidad, anticipar la vigilancia clínica y tener un mejor manejo de los recursos para el cuidado de los infantes en la Unidad de Medicina Familiar «lgnacio García Téllez» de Tepic, Nayarit.

\section{AGRADECIMIENTOS}

Externamos nuestro agradecimiento al Instituto Mexicano del Seguro Social por el apoyo recibido durante el periodo de especialidad en Medicina Familiar realizado en la Unidad de Medicina Familiar Núm. 24 «lgnacio García Téllez» de Tepic, Nayarit.

\section{Bibliografía}

1. Aguilar CM, Sánchez LA, Madrid BN, Mur VN, Expósito RM, Hermoso RE. Lactancia materna como prevención del sobrepeso y la obesidad en el niño y el adolescente: revisión sistemática. Nutr Hosp. 2015; 31 (2): 606-620.

2. Niño R, Silva G, Atalah E. Factores asociados a la lactancia materna exclusiva. Rev Chil Pediatr. 2012; 83 (2): 161-169.

3. Gutiérrez J, Rivera-Dommarco J, Shamah-Levy T, OropezaAbúndez C, Hernández Ávila M. Encuesta Nacional de Salud y Nutrición 2012, resultados por entidad federativa. Cuernavaca, Morelos, México: Instituto Nacional de Salud Pública; 2012. Disponible en: https://ensanut.insp.mx/ informes/Nayarit-OCT.pdf.

4. Hörnell A, Lagström H, Lande B, Thorsdottir I. Breastfeeding, introduction of other foods and effects on health: a systematic literature review for the 5th Nordic Nutrition Recommendations. Food Nutr Res. 2013; 57 (1): 21667.

5. Torrejón S, Osorio J, Vildoso F, Castillo C. Alimentación del niño menor de 2 años: recomendaciones de la Rama de Nutrición de la Sociedad Chilena de Pediatría. Rev Chil Pediatr. 2005; 76 (1): 97-97.

6. Osorio E, Weisstaub N, Castillo D. Desarrollo de la conducta alimentaria en la infancia y sus alteraciones. Rev Chil Nutr. 2002; 29 (3): 280-285.

7. Kramer MS, Kakuma R. Optimal duration of exclusive breastfeeding. Cochrane Database Syst Rev. 2012; (8): 1-42.

8. Shamah-Levy T, Cuevas-Nasu L, Gaona-Pineda EB, GómezAcosta LM, Morales-Ruán MC, Hernández-Ávila M et al. Sobrepeso y obesidad en niños y adolescentes en México, actualización de la Encuesta Nacional de Salud y Nutrición de Medio Camino 2016. Salud Publ Mex. 2018; 60 (3): 244253.

9. Camacho-Guerrero I, Rodríguez-Zepeda JJ, OswaldoSánchez E, Rodríguez-Arellano ME, Musalem-Younes C. Prevalence of obesity in preschoolers, students and adolescents at Hospital Regional Licenciado Adolfo López Mateos from ISSSTE. Rev Esp Med Quir. 2015; 20 (2): 152-157.

10. Wollenstein-Seligson D, Iglesias-Leboreiro J, BernárdezZapata I, Braverman-Bronstein A. Prevalencia de sobrepeso y obesidad infantil en un Hospital Privado de la Ciudad de México. Rev Mex Pediatr. 2016; 83 (4): 108-114.

11. Cu L, Villarreal E, Rangel B, Galicia L, Vargas E, Martínez L. Factores de riesgo para sobrepeso y obesidad en lactantes. Rev Chil Nutr. 2015;42(2):139-44.

12. Baidal JAW, Locks LM, Cheng ER, Blake-Lamb TL, Perkins ME, Taveras EM. Risk factors for childhood obesity in the first 1,000 days: a systematic review. Am J Prev Med. 2016; 50 (6): 761-779.

13. Larqué E, Labayen I, Flodmark CE, Lissau I, Czernin S, Moreno LA et al. From conception to infancy-early risk factors for childhood obesity. Nat Rev Endocrinol. 2019; 15 (8): 456-478.

14. Balaban G, Motta MEFA, Silva GAP. Early weaning and other potential risk factors for overweight among preschool children. Clinics. 2010; 65 (2): 187-187.

15. Onis M. WHO Child Growth Standards based on length/ height, weight and age. Acta Paediatr. 2006; 95 (S450): 76-85.

16. Morán RM, Naveiro RJ, Blanco FE, Cabañeros Al, Rodríguez FM, Peral CA. Prevalencia y duración de la lactancia materna: Influencia sobre el peso y la morbilidad. Nutr Hosp. 2009; 24 (2): 213-217.

17. Anzman SL, Rollins BY, Birch LL. Parental influence on children's early eating environments and obesity risk: implications for prevention. Int J Obes. 2010; 34 (7): 11161124.

18. Kim J, Peterson KE, Scanlon KS, Fitzmaurice GM, Must A, Oken E et al. Trends in overweight from 1980 through 
2001 among preschool-aged children enrolled in a health maintenance organization. Obesity. 2006 ; 14 (7): 1107-1112.

19. Reyes D, Reyes H, Sánchez C, Alonso C, Toledo M. Alimentos contenidos en loncheras de niños que acuden a preescolar. Bol Clin Hosp Infant Edo Son. 2010; 27 (1): 35-40.

20. Farrow C, Haycraft E, Mitchell G. Milk feeding, solid feeding, and obesity risk: a review of the relationships between early life feeding practices and later adiposity. Curr Obes Rep. 2013; 2 (1): 58-64.

21. Cosío-Martínez TG, Hernández-Cordero S, RiveraDommarco J, Hernández-Ávila M. Recomendaciones para una política nacional de promoción de la lactancia materna en México: postura de la Academia Nacional de Medicina. Salud Publ Mex. 2017; 59: 106-113.

\section{Anexo 1: Formato de cuestionario.}

Instituto Mexicano del Seguro Social

Unidad de Medicina Familiar Núm. 24 «lgnacio García Téllez»

Recolección de datos para determinar la prevalencia de sobrepeso y obesidad en lactantes mayores

Instrucciones: Lea cuidadosamente la oración y complete o elija la opción que usted considere que identifique a su hijo:

Peso al nacer

Nombre

Edad

Género

M

Procedencia

Peso actual

gramos

Talla $\mathrm{cm}$

IMC $\mathrm{kg} / \mathrm{m}^{2}$

Estado nutricional

Lactancia

Sí No

Seno materno

Fórmula láctea

Mixta

Duración meses

Inicio de alimentación complementaria mes

Inicio de alimentación complementaria con:

$\square$ Carne

$\square$ Frutas

Verduras

$\square$ Cereales

$\square$ Leguminosas

Derivados de la leche 